\title{
HARMONIC MEASURE AND HYPERBOLIC DISTANCE IN JOHN DISKS
}

\author{
KIWON KIM and NAVAH LANGMEYER
}

\section{Introduction.}

Suppose that $D$ is a domain in the complex plane C. Let $D^{*}=\mathrm{C} \backslash \bar{D}$ be the exterior of $D$ in $C$ and let $B(z, r)=\{\zeta:|\zeta-z|<r\}$ for $z \in \mathrm{C}$ and $r>0$.

In this paper, we find several characterizations of John disks which have analogues in the class of quasidisks. John disks can be thought of as "onesided quasidisks". For example, a Jordan domain $D \subset \mathrm{C}$ is a quasidisk if and only if $D$ and $D^{*}$ are John disks. Also, every quasidisk is a John disk [GM3]. The results presented here are likewise one-sided versions of characterizations of quasidisks. These characterizations involve the conformal invariants harmonic measure and hyperbolic distance.

A simply-connected bounded domain $D \subset \mathrm{C}$ is said to be a $c$-John disk if there exist a point $z_{0} \in D$ and a constant $c \geq 1$ such that each point $z_{1} \in D$ can be joined to $z_{0}$ by an arc $\gamma$ in $D$ satisfying

$$
\ell\left(\gamma\left(z_{1}, z\right)\right) \leq c(z, \partial D)
$$

for each $z \in \gamma$, where $\ell\left(\gamma\left(z_{1}, z\right)\right)$ is the euclidean length of the subarc of $\gamma$ with endpoints $z_{1}, z$. We call $z_{0}$ a John center, $c$ a John constant and $\gamma$ a $c$ John arc. We say that $D$ is John if it is $c$-John disk for some $c$.

A bounded domain $D \subset \mathrm{C}$ is John if and only if each pair of points $z_{1}, z_{2} \in D$ can be joined by an arc $\gamma$ which satisfies

$$
\min _{j=1,2} \ell\left(\gamma\left(z_{j}, z\right)\right) \leq c(z, \partial D)
$$

for all $z \in \gamma$. We call $\gamma$ a double c-cone arc. This definition can be used to define the unbounded John disks $D \subset \mathrm{C}$ as well [NV, 2.26].

A domain $D \subset \mathrm{C}$ is said to be $c$-uniform if there is a constant $c \geq 1$ such 
that each pair of points $z_{1}, z_{2} \in D$ can be joined by an arc $\gamma \subset D$ which satisfies (1.1) and

$$
\ell(\gamma) \leq c\left|z_{1}-z_{2}\right|
$$

We say that $D$ is uniform if it is $c$-uniform for some $c \geq 1$.

We say that a domain $D \subset \mathrm{C}$ is a $K$-quasidisk, $1 \leq K<\infty$, if it is the image of the unit disk B under a $K$-quasiconformal self mapping of $\overline{\mathrm{C}}=\mathrm{C} \cup \infty$. A Jordan domain $D \subset \mathrm{C}$ is uniform if and only if it is a quasidisk [MS].

In section 2, we show that a bounded Jordan domain $D \subset \mathrm{C}$ satisfies a harmonic doubling condition if and only if $D$ is a John disk. This is a onesided analogue of a characterization for quasidisks due to Jerison and Kenig [JK]. It is also a one-sided version of a characterization for quasidisks due to Krzyż who compares the harmonic measures of adjacent arcs on the boundary when considered from inside and outside the domain [Kr].

In section 3 , we characterize John disks $D$ in terms of various properties of the hyperbolic geodesics in $D$; in particular, the position of the euclidean midpoint of the geodesic or the quasiextremal distance property in $D$ with respect to the geodesic. The first of these leads to a third characterization in terms of the Hölder continuity of analytic functions in $D$ similar to a wellknown theorem of Hardy and Littlewood [HL]. Finally, we characterize unbounded Jordan John disks in terms of the hyperbolic geodesics in their exteriors.

In section 4, we characterize John disks in terms of a euclidean estimate for the hyperbolic distance between points of $D$. This is again a one-sided analogue of a theorem due to Gehring and Osgood [GO], who showed that a domain $D$ is uniform if and only if it satisfies

$$
k_{D}\left(z_{1}, z_{2}\right) \leq c j_{D}\left(z_{1}, z_{2}\right)+d
$$

for all $z_{1}, z_{2} \in D$ and some constants $c$ and $d$, where $k_{D}$ is the quasihyperbolic metric in $D$ and

$$
j_{D}\left(z_{1}, z_{2}\right)=\frac{1}{2} \log \left(\frac{\left|z_{1}-z_{2}\right|}{\operatorname{dist}\left(z_{1}, \partial D\right)}+1\right)\left(\frac{\left|z_{1}-z_{2}\right|}{\operatorname{dist}\left(z_{2}, \partial D\right)}+1\right) .
$$

Our result replaces the euclidean distance $\left|z_{1}-z_{2}\right|$ with the inner distance between these points and yields an analogous estimate for $h_{D}\left(z_{1}, z_{2}\right)$, the hyperbolic distance between $z_{1}$ and $z_{2}$.

We will repeatedly use a result of Gehring and Hayman.

Lemma 1.3 [GH, Theorem 2], [Ja]. Suppose that D is a simply connected domain in $\mathrm{C}$. If $\gamma$ is a hyperbolic geodesic in $D$ and if $\alpha$ is any curve which joins the endpoints of $\gamma$ in $D$, then 


$$
\ell(\gamma) \leq k \ell(\alpha)
$$

where $k$ is an absolute constant, $4.5 \leq k \leq 17.5$.

\section{Harmonic measure in John disks.}

A bounded Jordan domain $D \subset \mathrm{C}$ is said to satisfy a harmonic doubling condition if for some $z_{0} \in D$ and some constant $c_{0}>0$,

$$
\omega\left(z_{0}, \alpha ; D\right) \leq c_{0} \omega\left(z_{0}, \beta ; D\right)
$$

for each pair of consecutive $\operatorname{arcs} \alpha, \beta$ on $\partial D$ with $\operatorname{dia}(\alpha) \leq 2 \operatorname{dia}(\beta)$, where $\omega\left(z_{0}, \gamma ; D\right)$ is the harmonic measure of $\gamma$ at the point $z_{0}$ with respect to $D$.

Remark 2.2. If $D$ satisfies (2.1) for some $z_{0} \in D$, then it satisfies (2.1) for every $z_{1} \in D$ with a constant $c_{1}$ which depends on $c_{0}, z_{0}$ and $z_{1}$.

Proof. Fix $z_{1} \in D$ and fix consecutive $\operatorname{arcs} \alpha, \beta \subset \partial D$ with $\operatorname{dia}(\alpha) \leq$ $2 \operatorname{dia}(\beta)$. Since $\omega$ is nonnegative and harmonic,

$$
\frac{\omega\left(z_{1}, \alpha ; D\right)}{\omega\left(z_{0}, \alpha ; D\right)} \leq k \quad \text { and } \quad \frac{\omega\left(z_{0}, \beta ; D\right)}{\omega\left(z_{1}, \beta ; D\right)} \leq k,
$$

where $k=e^{h_{D}\left(z_{0}, z_{1}\right)}$. (See, for example, [H, Theorem 6].) Thus by hypothesis we have

$$
\frac{\omega\left(z_{1}, \alpha ; D\right)}{\omega\left(z_{1}, \beta ; D\right)} \leq \frac{\omega\left(z_{0}, \alpha ; D\right)}{\omega\left(z_{0}, \beta ; D\right)} k^{2} \leq c_{0} k^{2}=c_{1}
$$

and hence (2.1) holds for every $z_{1} \in D$ with $c_{1}=c_{0}\left(e^{h_{D}\left(z_{0}, z_{1}\right)}\right)^{2}$.

Theorem 2.3. A bounded Jordan domain $D \subset \mathrm{C}$ is a c-John disk if and only if it satisfies a harmonic doubling condition.

To prove Theorem 2.3 we need a lemma.

Lemma 2.4. Suppose that $D$ is a bounded Jordan domain in $\mathrm{C}$ and let $z_{0} \in D$. Then the following conditions are equivalent, where the constants in each condition need not be the same but depend on each other:

(1) D is a c-John disk.

(2) There exist constants $c$ and $\delta>0$ such that

$$
\frac{\operatorname{dia}\left(\alpha_{1}\right)}{\operatorname{dia}(\alpha)} \leq c\left(\frac{\omega\left(z_{0}, \alpha_{1} ; D\right)}{\omega\left(z_{0}, \alpha ; D\right)}\right)^{\delta}
$$

for all arcs $\alpha_{1} \subset \alpha \subset \partial D$.

(3) There exists a constant $c>1$ such that

$$
\omega\left(z_{0}, \alpha ; D\right) \leq c \omega\left(z_{0}, \alpha_{1} ; D\right)
$$


for all arcs $\alpha_{1} \subset \alpha \subset \partial D$ with $\operatorname{dia}(\alpha) \leq 2 \operatorname{dia}\left(\alpha_{1}\right)$.

Proof. The equivalence of (1) and (2) is proved in [P, Theorem 1]. To prove the equivalence of (2) and (3), we first assume that (2) holds and let $\alpha_{1} \subset \alpha$ be arcs on $\partial D$ with $\operatorname{dia}(\alpha) \leq 2 \operatorname{dia}\left(\alpha_{1}\right)$. Then

$$
\frac{\omega\left(z_{0}, \alpha_{1} ; D\right)}{\omega\left(z_{0}, \alpha ; D\right)} \geq c^{-\frac{1}{\delta}}\left(\frac{\operatorname{dia}\left(\alpha_{1}\right)}{\operatorname{dia}(\alpha)}\right)^{\frac{1}{\delta}} \geq(2 c)^{-\frac{1}{\delta}}
$$

and hence we have (2.6) with a constant $(2 c)^{\frac{1}{8}}$. Next suppose that (3) holds. Then by induction it is not difficult to show that

$$
\omega\left(z_{0}, \alpha ; D\right) \leq c^{n} \omega\left(z_{0}, \alpha_{1} ; D\right)
$$

for all arcs $\alpha_{1} \subset \alpha \subset \partial D$ with $\operatorname{dia}(\alpha) \leq 2^{n} \operatorname{dia}\left(\alpha_{1}\right)$ and for each integer $n>0$.

Now given any arcs $\alpha_{1} \subset \alpha \subset \partial D$, there exists an integer $n>0$ such that

$$
2^{n-1} \operatorname{dia}\left(\alpha_{1}\right) \leq \operatorname{dia}(\alpha) \leq 2^{n} \operatorname{dia}\left(\alpha_{1}\right) .
$$

Then by (2.7) we have

$$
\omega\left(z_{0}, \alpha ; D\right) \leq c^{n} \omega\left(z_{0}, \alpha_{1} ; D\right) .
$$

Let $\delta=\frac{\log 2}{\log c}$. Then by (2.8) and (2.9) we obtain

$$
\frac{\omega\left(z_{0}, \alpha ; D\right)}{\omega\left(z_{0}, \alpha_{1} ; D\right)} \leq c^{n}=c\left(2^{\frac{1}{\delta}}\right)^{n-1}=c\left(2^{n-1}\right)^{\frac{1}{\delta}} \leq c\left(\frac{\operatorname{dia}(\alpha)}{\operatorname{dia}\left(\alpha_{1}\right)}\right)^{\frac{1}{\delta}} .
$$

Hence we get (2.5) with a constant $c^{\delta}$.

Proof of Theorem 2.3. For the necessity suppose that a harmonic doubling condition does not hold for $D$. Then for $j=1,2, \ldots$ there are consecutive arcs $\alpha_{j}, \beta_{j}$ on $\partial D$ such that

$$
\operatorname{dia}\left(\alpha_{j}\right) \leq 2 \operatorname{dia}\left(\beta_{j}\right) \quad \text { and } \quad \omega\left(z_{0}, \alpha_{j} ; D\right) \geq 3^{j} \omega\left(z_{0}, \beta_{j} ; D\right) .
$$

Thus $\operatorname{dia}\left(\alpha_{j} \cup \beta_{j}\right) \leq 3 \operatorname{dia}\left(\beta_{j}\right)$ and hence by Lemma 2.4 (2) and by (2.10)

$$
\frac{1}{3} \leq \frac{\operatorname{dia}\left(\beta_{j}\right)}{\operatorname{dia}\left(\alpha_{j} \cup \beta_{j}\right)} \leq c\left(\frac{\omega\left(z_{0}, \beta_{j} ; D\right)}{\omega\left(z_{0}, \alpha_{j} \cup \beta_{j} ; D\right)}\right)^{\delta} \leq c\left(\frac{\omega\left(z_{0}, \beta_{j} ; D\right)}{\omega\left(z_{0}, \alpha_{j} ; D\right)}\right)^{\delta} \leq c\left(3^{-j}\right)^{\delta}
$$

which yields a contradiction as $j \rightarrow \infty$.

For the sufficiency, by Lemma 2.4 it suffices to show that $D$ satisfies (2.6). Let $\alpha_{1} \subset \alpha$ be arcs of $\partial D$ with $\operatorname{dia}(\alpha) \leq 2 \operatorname{dia}\left(\alpha_{1}\right)$.

Suppose first that $\alpha_{1}, \alpha$ have a common endpoint. Then $\operatorname{dia}\left(\alpha \backslash \alpha_{1}\right) \leq$ $2 \operatorname{dia}\left(\alpha_{1}\right)$ and hence by (2.1), $\omega\left(z_{0}, \alpha \backslash \alpha_{1} ; D\right) \leq c_{0} \omega\left(z_{0}, \alpha_{1} ; D\right)$ for some $z_{0} \in D$. Thus 


$$
\omega\left(z_{0}, \alpha ; D\right) \leq\left(c_{0}+1\right) \omega\left(z_{0}, \alpha_{1} ; D\right) .
$$

Next suppose that $\alpha \backslash \alpha_{1}$ consists of two disjoint subarcs $\alpha_{2}, \alpha_{3}$. Then for $j=2,3 \quad \operatorname{dia}\left(\alpha_{1} \cup \alpha_{j}\right) \leq 2 \operatorname{dia}\left(\alpha_{1}\right)$ and hence $\omega\left(z_{0}, \alpha_{1} \cup \alpha_{j} ; D\right) \leq\left(c_{0}+1\right)$ $\omega\left(z_{0}, \alpha_{1} ; D\right)$ by what was proved above. Thus

$$
\omega\left(z_{0}, \alpha ; D\right) \leq 2\left(c_{0}+1\right) \omega\left(z_{0}, \alpha_{1} ; D\right) .
$$

Therefore by (2.11) and (2.12) $D$ satisfies (2.6) with $c=2\left(c_{0}+1\right)$.

\section{Hyperbolic geodesics in John disks.}

We say that a domain $D \subset \mathrm{C}$ is a $M$-quasiextremal distance or $M$-QED domain with respect to $E \subset D, 1 \leq M<\infty$, if for each pair of disjoint continua $F_{1}, F_{2} \subset E$

$$
\bmod (\Gamma) \leq M \bmod \left(\Gamma_{D}\right)
$$

where $\Gamma$ and $\Gamma_{D}$ are the families of curves joining $F_{1}$ and $F_{2}$ in $\mathrm{C}$ and in $D$, respectively.

Theorem 3.2. Suppose that D is a bounded simply connected domain in $\mathrm{C}$. Then the followings are equivalent:

(1) D is a c-John disk.

(2) There exists a constant $c>0$ such that for each hyperbolic geodesic $\gamma \subset D$

$$
\ell(\gamma) \leq c\left(z_{0}, \partial D\right)
$$

where $z_{0}$ is the euclidean midpoint of $\gamma$.

(3) There exists a constant $c>0$ such that if $f$ is analytic with

$$
\left|f^{\prime}(z)\right| \leq 1
$$

in $D$, then for all $z_{1}, z_{2} \in D$

$$
\left|f\left(z_{1}\right)-f\left(z_{2}\right)\right| \leq c \operatorname{dist}\left(z_{0}, \partial D\right),
$$

where $z_{0}$ is the euclidean midpoint of the hyperbolic geodesic $\gamma \subset D$ joining $z_{1}$ to $z_{2}$.

(4) $D$ is a $M$-QED domain with respect to all hyperbolic geodesics in $D$ with a given point $z_{0} \in D$ as an endpoint.

Here the constants in each condition need not be the same but depend on each other. In particular, from (4) we obtain a John constant $c$ in (1), which depends on $M$ and a given point $z_{0}$.

Proof of equivalence of (1) AND (2). Let $D \subset \mathrm{C}$ be a bounded $c$-John disk and let $z_{0}$ be the euclidean midpoint of a hyperbolic geodesic $\gamma$ with 
endpoints $z_{1}$ and $z_{2}$ in $D$. By [GHM, 2.16 Lemma], there exists a crosscut $\alpha$ of $D$ containing $z_{0}$ which separates the components of $\gamma \backslash\left\{z_{0}\right\}$ in $D$ and

$$
\ell(\alpha) \leq c_{1} \operatorname{dist}\left(z_{0}, \partial D\right),
$$

where $c_{1}$ is an absolute constant. Next since $D$ is a $c$-John disk, there exists a John center $x_{0}$, a c-John arc $\beta_{1}$ from $z_{1}$ to $x_{0}$, and a $c$-John arc $\beta_{2}$ from $z_{2}$ to $x_{0}$.

If $x_{0}$ is in the component of $D \backslash \alpha$ which contains $z_{2}$, then by (3.6) there exists a point $w$ in $\alpha \cap \beta_{1}$ such that

$$
\ell\left(\beta_{1}\left(z_{1}, w\right)\right) \leq c \operatorname{dist}(w, \partial D) \leq c \ell(\alpha) \leq c c_{1} \operatorname{dist}\left(z_{0}, \partial D\right) .
$$

Since $\beta_{1}\left(z_{1}, w\right) \cup \alpha\left(z_{0}, w\right)$ is a curve which joins $z_{1}$ to $z_{0}$ in $D$ and since $\gamma\left(z_{1}, z_{0}\right)$ is a hyperbolic geodesic in $D$ with $z_{1}$ and $z_{0}$ as its end points, Lemma 1.3 and (3.6) imply that

$\ell(\gamma)=2 \ell\left(\gamma\left(z_{1}, z_{0}\right)\right) \leq 2 k\left(\ell\left(\beta_{1}\left(z_{1}, w\right)\right)+\ell\left(\alpha\left(z_{0}, w\right)\right)\right)=2 k c_{1}(c+1) \operatorname{dist}\left(z_{0}, \partial D\right)$

where $k$ is an absolute constant. If $x_{0}$ is in the component of $D \backslash \alpha$ which contains $z_{1}$, then the above argument applied to the arc $\beta_{2}$ yields the desired inequality. Finally if $x_{0} \in \alpha$, then by Lemma 1.3 and (3.6),

$$
\begin{aligned}
\ell(\gamma) & =2 \ell\left(\gamma\left(z_{1}, z_{0}\right)\right) \leq 2 k\left(\ell\left(\beta_{1}\right)+\ell\left(\alpha\left(x_{0}, z_{0}\right)\right)\right) \\
& \leq 2 k\left(c \operatorname{dist}\left(x_{0}, \partial D\right)+c_{1} \operatorname{dist}\left(z_{0}, \partial D\right)\right) .
\end{aligned}
$$

Since $\alpha$ joins $x_{0}$ to $\partial D$,

$$
\ell(\gamma) \leq 2 k\left(c \ell(\alpha)+c_{1} \operatorname{dist}\left(z_{0}, \partial D\right)\right) \leq 2 k c_{1}(c+1) \operatorname{dist}\left(z_{0}, \partial D\right) .
$$

Conversely, suppose that (2) holds and let $L=\sup \ell(\gamma)$, where the supremum is taken over all possible hyperbolic geodesics $\gamma$ with endpoints in $D$. Then there exist two points $z_{1}, z_{2} \in D$ such that $\ell(\gamma)=\frac{L}{2}$, where $\gamma$ is the hyperbolic geodesic joining $z_{1}$ to $z_{2}$ in $D$. Let $z_{0}$ be the euclidean midpoint of $\gamma$. Then by (3.3),

$$
\operatorname{dia}(D) \geq \operatorname{dist}\left(z_{0}, \partial D\right) \geq \frac{1}{c} \ell(\gamma)=\frac{1}{2 c} L .
$$

Now fix a point $z \in D$ and let $w_{0}$ be the euclidean midpoint of the hyperbolic geodesic $\alpha$ joining $z$ to $z_{0}$ in $D$. If $x \in \alpha\left(w_{0}, z\right)$, then we can find a point $x_{1} \in \alpha\left(x, z_{0}\right)$ with $\ell(\alpha(z, x))=\ell\left(\alpha\left(x, x_{1}\right)\right)$ and by (3.3) applied to $\alpha\left(z, x_{1}\right)$,

$$
\ell(\alpha(z, x))=\frac{1}{2} \ell\left(\alpha\left(z, x_{1}\right)\right) \leq \frac{c}{2} \operatorname{dist}(x, \partial D) .
$$

If $x \in \alpha\left(z_{0}, w_{0}\right)$, then we can find a point $x_{2} \in \alpha(x, z)$ with $\ell\left(\alpha\left(x, z_{0}\right)\right)=$ $\ell\left(\alpha\left(x_{2}, x\right)\right)$. Then again by (3.3) applied to $\alpha\left(z_{0}, x_{2}\right)$ and by (3.7), 


$$
\begin{aligned}
\ell(\alpha(z, x)) & \leq L \leq 2 c \operatorname{dist}\left(z_{0}, \partial D\right) \leq 2 c\left(\ell\left(\alpha\left(x_{2}, z_{0}\right)\right)+\operatorname{dist}(x, \partial D)\right) \\
& \leq 2 c(c+1) \operatorname{dist}(x, \partial D) .
\end{aligned}
$$

Hence by (3.8) and (3.9) D is a $c_{1}$-John disk with $c_{1}=2 c(c+1)$.

Proof OF eQuivalence of (2) AND (3). First suppose that $D$ satisfies (2). Then $D$ is a $b$-John disk, where $b$ depends only on $c$. Let $f$ be analytic and satisfy

$$
\left|f^{\prime}(z)\right| \leq \operatorname{dist}(z, \partial D)^{\alpha-1}
$$

for some $0<\alpha \leq 1$ in $D$. Fix $z_{1}, z_{2} \in D$, and let $\gamma$ be the hyperbolic geodesic joining $z_{1}$ to $z_{2}$ in $D$. Next let $s$ denote arclength measured along $\gamma$ from $z_{1}$, let $z(s)$ denote the corresponding representation for $\gamma$, and set $g(s)=f(z(s))$. Then

$$
\left|g^{\prime}(s)\right|=\left|f^{\prime}(z(s))\right|
$$

while

$$
\min (s, l-s) \leq b_{1} \operatorname{dist}(z(s), \partial D), \quad l=\ell(\gamma),
$$

where $b_{1} \geq 1$ is a constant depending only on $b$, by [GHM, Theorem 4.1]. Thus

$$
\left|g^{\prime}(s)\right| \leq \operatorname{dist}(z(s), \partial D)^{\alpha-1} \leq\left(\frac{\min (s, l-s)}{b_{1}}\right)^{\alpha-1}
$$

for $0<s<l$, and hence

$$
\begin{aligned}
\left|f\left(z_{1}\right)-f\left(z_{2}\right)\right| & =|g(l)-g(0)| \\
& \leq \int_{0}^{l}\left|g^{\prime}(s)\right| d s \leq 2 b_{1}{ }^{1-\alpha} \int_{0}^{\frac{l}{2}} s^{\alpha-1} d s \\
& =\frac{2 b_{1}{ }^{1-\alpha}}{\alpha}\left(\frac{l}{2}\right)^{\alpha} \leq \frac{c_{1}}{\alpha} \operatorname{dist}\left(z_{0}, \partial D\right)^{\alpha},
\end{aligned}
$$

where $c_{1}=b_{1} c$. If $f$ satisfies (3.4) in $D$, then $f$ satisfies (3.10) with $\alpha=1$. Hence, $f$ satisfies (3.11) with $\alpha=1$, i.e. $f$ satisfies (3.5).

Now suppose that (3.5) holds for any analytic function $f$ on $D$ which satisfies (3.4). By [KW, Theorem 1] with $k=1$, for $z_{1}, z_{2} \in D$

$$
\inf _{\beta} \int_{\beta}|d \zeta| \leq c_{1} \sup _{f}\left|f\left(z_{1}\right)-f\left(z_{2}\right)\right|,
$$

where the infimum is taken over all Jordan $\operatorname{arcs} \beta$ in $D$ joining $z_{1}$ to $z_{2}, c_{1}$ is 
an absolute constant, and the supremum is taken over all analytic functions $f$ on $D$ with $\left|f^{\prime}(z)\right| \leq 1$. Thus by Lemma 1.3,

$$
\ell(\gamma) \leq k \inf _{\beta} \ell(\beta) \leq k c_{1} \sup _{f}\left|f\left(z_{1}\right)-f\left(z_{2}\right)\right| \leq k c_{1} c \operatorname{dist}\left(z_{0}, \partial D\right)
$$

for an absolute constant $k$, where $\gamma$ is the hyperbolic geodesic joining $z_{1}$ to $z_{2}$ in $D$ and $z_{0}$ is the euclidean midpoint of $\gamma$.

REMARK 3.12. Note that this proof shows that if (3.4) implies (3.5), then $D$ satisfies (2) and hence

$$
\left|f^{\prime}(z)\right| \leq \operatorname{dist}(z, \partial D)^{\alpha-1}
$$

in $D$ implies

$$
\left|f\left(z_{1}\right)-f\left(z_{2}\right)\right| \leq \frac{c}{\alpha} \operatorname{dist}\left(z_{0}, \partial D\right)^{\alpha}
$$

for any $0<\alpha \leq 1$.

In order to prove the equivalence of (1) and (4), we need a lemma which shows that each hyperbolic line in $D$ which joins two points on $\partial D$ lies in the middle of $D$. See [R, Lemma 4.13] and [PR, Theorem 3.3].

Lemma 3.13. Suppose that $D$ is a simply connected proper subdomain in $\mathrm{C}$ and that $\gamma \subset D$ is a hyperbolic line joining $w_{1}, w_{2} \in \partial D$ and dividing $D$ into disjoint subdomains $D_{1}$ and $D_{2}$. Then

$$
\frac{1}{b} \leq \frac{\operatorname{dist}\left(z, \alpha_{1}\right)}{\operatorname{dist}\left(z, \alpha_{2}\right)} \leq b, \quad b=3+2 \sqrt{2}
$$

for all $z \in \gamma$, where $\alpha_{j}=\partial D_{j} \backslash \bar{\gamma}, j=1,2$.

Proof of Equivalence of (1) AND (4). Suppose that $D \subset \mathrm{C}$ is a bounded $c$-John disk with fixed John center $z_{0}$. Fix $z_{1} \in D$ and let $\gamma$ be the hyperbolic geodesic joining $z_{0}$ to $z_{1}$ in $D$. Fix two disjoint continua $F_{1}, F_{2}$ of $\gamma$. Then by [K, Theorem 2.1] and the construction on [GO, pp. 67-68], there is a $K$ quasidisk $G_{1}$ in $D$ such that $\gamma \in \overline{G_{1}}$, where $K$ depends only on $c$. Thus by [GM3, Remark 2.23], $G_{1}$ is $M$-QED with respect to $G_{1}$ for some constant $M$, $1 \leq M<\infty$, which depends only on $K$, and hence only on $c$. Therefore, since $\Gamma_{G_{1}} \subset \Gamma_{D}$,

$$
\bmod (\Gamma) \leq M \bmod \left(\Gamma_{G_{1}}\right) \leq M \bmod \left(\Gamma_{D}\right),
$$

where $\Gamma, \Gamma_{G_{1}}, \Gamma_{D}$ are the families of curves which join $F_{1}$ and $F_{2}$ in $\mathrm{C}, G_{1}, D$, respectively.

Suppose next that $z_{0}$ is a point in $D$ and that $D$ is $M$-QED with respect to 
all hyperbolic geodesics in $D$ which have $z_{0}$ as an endpoint. Fix $z_{1} \in D$, $z_{1} \neq z_{0}$ and let $\gamma$ be the hyperbolic geodesic joining $z_{0}$ to $z_{1}$ in $D$.

We show first that for some constant $a>1$ and for all $z \in \gamma$

$$
\min \left(\left|z_{0}-z\right|,\left|z-z_{1}\right|\right) \leq a \operatorname{dist}(z, \partial D) .
$$

Suppose otherwise. Then for each constant $a>1$, there is a point $z \in \gamma$ such that $\min \left(\left|z_{0}-z\right|,\left|z-z_{1}\right|\right)>a \operatorname{dist}(z, \partial D)$. Fix a constant $a>1$ and let $b=3+2 \sqrt{2}$. Then for a constant $a b>1$ there is a point $z \in \gamma$ such that

$$
\min \left(\left|z_{0}-z\right|,\left|z-z_{1}\right|\right)>a b \operatorname{dist}(z, \partial D) .
$$

Consider the hyperbolic line in $D$ which contains $\gamma$ and which has the endpoints $w_{1}, w_{2} \in \partial D$ and let $\alpha_{1}, \alpha_{2}$ be as described in Lemma 3.13. Then $\operatorname{dist}(z, \partial D)=\min _{j=1,2} \operatorname{dist}\left(z, \alpha_{j}\right)$. Thus we may assume that $\operatorname{dist}(z, \partial D)=$ $\operatorname{dist}\left(z, \alpha_{1}\right)$ and hence by Lemma 3.13

$$
\operatorname{dist}\left(z, \alpha_{2}\right) \leq b \operatorname{dist}(z, \partial D)
$$

Let $r=b \operatorname{dist}(z, \partial D)$. By means of a preliminary similarity mapping we may assume that $z=0$. Then $z_{0}, z_{1} \notin B(0, a r)$. Let $A=B(0, a r) \backslash \bar{B}(0, \sqrt{a} r)$. For $j=0,1$, let $F_{j}$ denote a component of $A \cap \gamma\left(0, z_{j}\right)$ which joins the two boundary circles of $A$. Then by [V, Theorem 10.12],

$$
\bmod (\Gamma) \geq \bmod \left(\Gamma_{A}\right)=\frac{2}{\pi} \log \sqrt{a},
$$

where $\Gamma, \Gamma_{A}$ are the families of curves joining $F_{0}$ and $F_{1}$ in $\mathrm{C}$ and in $A$, respectively. Now let $B=B(0, \sqrt{a} r) \backslash \bar{B}(0, r), E=\partial B(0, r)$, and $F=\partial B(0, \sqrt{a} r)$. Then by (3.15), $\Gamma_{D}$ is minorized by $\Gamma_{B}$ and hence by $[\mathrm{V}, 7.5]$

$$
\bmod \left(\Gamma_{D}\right) \leq \bmod \left(\Gamma_{B}\right)=2 \pi\left(\log \frac{\sqrt{a} r}{r}\right)^{-1}=\frac{2 \pi}{\log \sqrt{a}}
$$

where $\Gamma_{B}$ is the family of curves joining $E$ and $F$ in $B$ and $\Gamma_{D}$ is the family of curves joining $F_{0}$ and $F_{1}$ in $D$. Then by the hypothesis, (3.16) and (3.17)

$$
\frac{2}{\pi} \log \sqrt{a} \leq \bmod (\Gamma) \leq M \bmod \left(\Gamma_{D}\right) \leq \frac{2 \pi M}{\log \sqrt{a}}
$$

and hence

$$
M \geq\left(\frac{\log \sqrt{a}}{\pi}\right)^{2} .
$$

This holds for each constant $a>1$ and it leads a contradiction, which establishes (3.14). 
Next to show that $D$ is a $c$-John disk, by [NV, Lemma 2.10] we need to prove that for some constant $c \geq 1$ and for all $z \in \gamma$

$$
\left|z-z_{1}\right|<c \operatorname{dist}(z, \partial D) \text {. }
$$

For this let $L=\max \left\{\left|z_{0}-z\right|: z \in \partial D\right\}, k=\frac{L}{\operatorname{dist}\left(z_{0}, \partial D\right)}$ and $c_{1}=\max (a, k)$. If $\left|z-z_{1}\right|<\left|z-z_{0}\right|$, then by (3.14)

$$
\left|z-z_{1}\right|<a \operatorname{dist}(z, \partial D) \text {. }
$$

If $\left|z-z_{1}\right|>\left|z-z_{0}\right|$, then $\left|z_{0}-z_{1}\right| \leq L$ and (3.14) give

$$
\begin{aligned}
\frac{\left|z-z_{1}\right|}{c_{1}} & <\frac{\left|z-z_{0}\right|}{a}+\frac{\left|z_{0}-z_{1}\right|}{k}<\operatorname{dist}(z, \partial D)+\operatorname{dist}\left(z_{0}, \partial D\right) \\
& \leq\left|z-z_{0}\right|+2 \operatorname{dist}(z, \partial D)<(a+2) \operatorname{dist}(z, \partial D) .
\end{aligned}
$$

Hence

$$
\left|z-z_{1}\right|<c_{1}(a+2) \operatorname{dist}(z, \partial D) \text {. }
$$

Therefore by (3.19) and (3.20) we obtain (3.18) with $c=c_{1}(a+2)$, which depends on $M$ and $z_{0}$.

Note that in the proof of equivalence of (1) and (4) in Theorem 3.2, what we get from (4) is the John condition on all hyperbolic geodesics with a given point $z_{0}$ as an end point and a fixed constant $c=c\left(z_{0}, M\right)$. If $c$ were independent of $z_{0}$, we are in the uniform domain case as follows.

Corollary 3.21. Suppose that $D$ is a bounded finitely connected domain in C. Then $D$ is c-uniform if and only if $D$ is a $M$-QED domain with respect to all hyperbolic geodesics in $D$. Here $c$ and $M$ depend only on each other.

Proof. Suppose that $D$ is $c$-uniform. Then by [GM3, Theorem 2.22], $D$ is a $M$-QED domain with respect to $D, M=M(c)$, and hence with respect to all hyperbolic geodesics in $D$. For the sufficiency, let $z_{1}, z_{2}$ be two disjoint points in $D$ and let $\gamma$ be the hyperbolic geodesic in $D$ with endpoints $z_{1}, z_{2}$. Then by an argument similar to that for the proof of (3.14)

$$
\min \left(\left|z_{1}-z\right|,\left|z-z_{2}\right|\right) \leq a \operatorname{dist}(z, \partial D)
$$

for all $z \in \gamma$ and for some constant $a>1$. Also by the same argument as the proof of [GM3, Lemma 2.7]

$$
\ell(\gamma) \leq k\left|z_{1}-z_{2}\right|,
$$

where $k, 1<k<\infty$, is a constant depending only on $M$. Therefore [NV, Theorem 2.16], (3.22) and (3.23) imply that $D$ is $c$-uniform with $c=\max (a, k)$, which depends only on $M$. 
Next we characterize unbounded Jordan John disks with $\infty \in \partial D$ in terms of the hyperbolic geodesics in their exteriors.

Lemma 3.24 [GHM], [NV], [R]. A Jordan domain D $\subset \mathrm{C}$ is a c-John disk if and only if each pair of points $z_{1}, z_{2} \in D^{*}$ can be joined by a continuum $E \subset D^{*}$ with

$$
\operatorname{dia}(E) \leq c_{1}\left|z_{1}-z_{2}\right|
$$

Here the constants $c$ and $c_{1}$ depend only on each other.

Theorem 3.25. A Jordan domain $D \subset \mathrm{C}$ with $\infty \in \partial D$ is a c-John disk if and only there is a constant $c_{0} \geq 1$ such that for each hyperbolic geodesic $\gamma$ in $D^{*}$

$$
\operatorname{dia}(\gamma) \leq c_{0}\left|z_{1}-z_{2}\right|
$$

where $z_{1}, z_{2}$ are the endpoints of $\gamma$. Here $c$ and $c_{0}$ depend on each other.

To prove this we need a lemma which gives the diameter version of the Gehring-Hayman inequality in Lemma 1.3. See [R, Lemma 3.22] and [PR, Theorem 3.2].

Lemma 3.27. Suppose that $\gamma$ is a hyperbolic geodesic in a simply connected proper subdomain $D \subset \mathrm{C}$ and that $\alpha$ is an arc which joins the endpoints of $\gamma$ in $D \cap \bar{B}\left(z_{0}, r\right)$ for $z_{0} \in$ C. Then

$$
\gamma \subset \bar{B}\left(z_{0}, b r\right), \quad b=3+2 \sqrt{2} .
$$

Proof of Theorem 3.25. Suppose first that a Jordan domain $D \subset \mathrm{C}$ is a $c$-John disk with $\infty \in \partial D$. Then by Lemma 3.24 for each pair of points $z_{1}, z_{2} \in D^{*}$ there exists a continuum $E \subset D^{*}$ such that $\operatorname{dia}(E) \leq c_{1}\left|z_{1}-z_{2}\right|$. Thus by [NV, Lemma 4.3], $E$ can be replaced by an arc $\alpha \subset D^{*}$ with $\operatorname{dia}(\alpha) \leq c_{2}\left|z_{1}-z_{2}\right|$ for any $c_{2}>c_{1}$. Next let $\gamma$ be the hyperbolic geodesic joining $z_{1}$ and $z_{2}$ in $D^{*}$. Then $\alpha$ is an arc which joins the endpoints of $\gamma$. Now choose a point $z_{0} \in \alpha$ such that $\left|z_{1}-z_{0}\right|=\left|z_{2}-z_{0}\right|$ and let $r=\operatorname{dia}(\alpha)$. Thus $\alpha \subset D^{*} \cap \bar{B}\left(z_{0}, r\right)$, while $\gamma \subset \bar{B}\left(z_{0}, b r\right)$ with $b=3+2 \sqrt{2}$ by Lemma 3.27. Hence

$$
\operatorname{dia}(\gamma) \leq 2 b r=2 b \operatorname{dia}(\alpha) \leq 2 b c_{2}\left|z_{1}-z_{2}\right|
$$

and this shows (3.26) with $c_{0}=2 b c_{2}$.

Suppose next that (3.26) holds. Then by Lemma 3.24, $D$ is a $c$-John disk. 


\section{Hyperbolic distance in John disks.}

We define a one-sided analogue of the function $j_{D}$ in (1.2) as follows:

$$
j_{D}^{\prime}\left(z_{1}, z_{2}\right)=\frac{1}{2} \log \left(\frac{\lambda_{D}\left(z_{1}, z_{2}\right)}{\operatorname{dist}\left(z_{1}, \partial D\right)}+1\right)\left(\frac{\lambda_{D}\left(z_{1}, z_{2}\right)}{\operatorname{dist}\left(z_{2}, \partial D\right)}+1\right),
$$

where $\lambda_{D}$ is the inner distance on $D$,

$$
\lambda_{D}\left(z_{1}, z_{2}\right)=\inf _{\gamma} \ell(\gamma),
$$

and the infimum is taken over all paths $\gamma \subset D$ with $z_{1}$ and $z_{2}$ as endpoints. The main result of this section relates $h_{D}$ and $j_{D}^{\prime}$ in John disks. As mentioned in the introduction, this is a one-sided analogue of a characterization of quasidisks due to Gehring and Osgood [GO]. Their two-sided version characterizes uniform domains, regardless of connectivity, when the hyperbolic metric is replaced by the quasihyperbolic metric.

THEOREM 4.1. A simply connected proper subdomain $D \subset \mathrm{C}$ is a c-John disk if and only if there exists a constant $b \geq 1$ such that

$$
h_{D}\left(z_{1}, z_{2}\right) \leq b j_{D}^{\prime}\left(z_{1}, z_{2}\right)
$$

for all $z_{1}, z_{2} \in D$. Here the constants $c$ and $b$ depend only on each other.

We will use the following inequality, which is easily derived.

Lemma 4.3. For any $c \geq 1$ and $x \geq 0$,

$$
\log (c x+1) \leq c \log (x+1) .
$$

Proof of NeCESSITY. Suppose that $D$ is a $c$-John disk. Then by [GHM, Theorem 4.1] each $z_{1}, z_{2} \in D$ can be joined by a hyperbolic geodesic $\gamma$ in $D$ such that for all $z \in \gamma$

$$
\min _{j=1,2} \ell\left(\gamma\left(z_{j}, z\right)\right) \leq c_{1} \operatorname{dist}(z, \partial D)
$$

for some constant $c_{1}$ depending only on $c$. Choose $z_{0} \in \gamma$ so that $\ell\left(\gamma\left(z_{0}, z_{1}\right)\right)=\ell\left(\gamma\left(z_{0}, z_{2}\right)\right)$. Then by the triangle inequality it is sufficient to show that

$$
h_{D}\left(z_{j}, z_{0}\right) \leq b \log \left(\frac{\lambda_{D}\left(z_{1}, z_{2}\right)}{\operatorname{dist}\left(z_{j}, \partial D\right)}+1\right)
$$

for $j=1,2$, where $b=\left(3 c_{1}+2\right) k$ and $k$ is an absolute constant. By symmetry we may assume that $j=1$.

Suppose first that 


$$
\ell\left(\gamma\left(z_{1}, z_{0}\right)\right) \leq \frac{c_{1}}{c_{1}+1} \operatorname{dist}\left(z_{1}, \partial D\right) .
$$

Then $z_{0} \in B\left(z_{1}, \frac{c_{1}}{c_{1}+1} \operatorname{dist}\left(z_{1}, \partial D\right)\right)$. If $z \in\left[z_{1}, z_{0}\right]$, then

$$
\operatorname{dist}(z, \partial D) \geq \operatorname{dist}\left(z_{1}, \partial D\right)-\left|z_{1}-z\right| \geq \frac{1}{c_{1}+1} \operatorname{dist}\left(z_{1}, \partial D\right)
$$

and hence

$$
\begin{aligned}
\left|z_{1}-z\right| & +\operatorname{dist}\left(z_{1}, \partial D\right) \leq c_{1} \operatorname{dist}(z, \partial D)+\left(c_{1}+1\right) \operatorname{dist}(z, \partial D) \\
& \leq\left(2 c_{1}+1\right) \operatorname{dist}(z, \partial D) .
\end{aligned}
$$

If $\rho_{D}(z)$ is the hyperbolic density in $D$, then the Schwarz lemma and the Koebe distortion theorem give the inequalities

$$
\frac{1}{4 \operatorname{dist}(z, \partial D)} \leq \rho_{D}(z) \leq \frac{1}{\operatorname{dist}(z, \partial D)} .
$$

Thus Lemma 1.3 and Lemma 4.3 yield

$$
\begin{aligned}
h_{D}\left(z_{1}, z_{0}\right) & \leq \int_{\left[z_{1}, z_{0}\right]} \frac{d s}{\operatorname{dist}(z, \partial D)} \\
& \leq \int_{0}^{\left|z_{1}-z_{0}\right|} \frac{\left(2 c_{1}+1\right) d s}{s+\operatorname{dist}\left(z_{1}, \partial D\right)} \\
& \leq\left(2 c_{1}+1\right) \log \left(\frac{\ell(\gamma)}{\operatorname{dist}\left(z_{1}, \partial D\right)}+1\right) \\
& \leq\left(2 c_{1}+1\right) k \log \left(\frac{\lambda_{D}\left(z_{1}, z_{2}\right)}{\operatorname{dist}\left(z_{1}, \partial D\right)}+1\right) .
\end{aligned}
$$

where $k$ is an absolute constant. This implies (4.5).

Next suppose that (4.6) does not hold and choose $y_{1} \in \gamma\left(z_{1}, z_{0}\right)$ so that

$$
\ell\left(\gamma\left(z_{1}, y_{1}\right)\right)=\frac{c_{1}}{c_{1}+1} \operatorname{dist}\left(z_{1}, \partial D\right)
$$

If $z \in \gamma\left(y_{1}, z_{0}\right)$, then

$$
\operatorname{dist}(z, \partial D) \geq \frac{1}{c_{1}} \ell\left(\gamma\left(z_{1}, z\right)\right)
$$

by (4.4) and hence again 


$$
\begin{aligned}
h_{D}\left(y_{1}, z_{0}\right) & \leq c_{1} \log \left(\frac{c_{1}+1}{c_{1}} \frac{\ell\left(\gamma\left(z_{1}, z_{0}\right)\right)}{\operatorname{dist}\left(z_{1}, \partial D\right)}\right) \\
& \leq c_{1} \log \left(\frac{c_{1}+1}{c_{1}} \frac{\ell(\gamma)}{\operatorname{dist}\left(z_{1}, \partial D\right)}+1\right) \\
& \leq\left(c_{1}+1\right) k \log \left(\frac{\lambda_{D}\left(z_{1}, z_{2}\right)}{\operatorname{dist}\left(z_{1}, \partial D\right)}+1\right) .
\end{aligned}
$$

We also have

$$
h_{D}\left(z_{1}, y_{1}\right) \leq\left(2 c_{1}+1\right) k \log \left(\frac{\lambda_{D}\left(z_{1}, y_{1}\right)}{\operatorname{dist}\left(z_{1}, \partial D\right)}+1\right)
$$

by what was proved above. Then (4.5) follows from the triangle inequality.

Proof of Sufficiency. Suppose that (4.2) holds. Fix $z_{1}, z_{2} \in D$ and let $\gamma$ be the hyperbolic geodesic joining $z_{1}$ to $z_{2}$ in $D$. We may assume that $\operatorname{dist}\left(z_{1}, \partial D\right) \geq \operatorname{dist}\left(z_{2}, \partial D\right)$.

Suppose first that

$$
2 \lambda_{D}\left(z_{1}, z_{2}\right) \leq \operatorname{dist}\left(z_{1}, \partial D\right)
$$

Then $\left|z_{1}-z_{2}\right| \leq \operatorname{dist}\left(z_{1}, \partial D\right) / 2$ and hence

$$
z_{2} \in B\left(z_{1}, \frac{\operatorname{dist}\left(z_{1}, \partial D\right)}{2}\right) \subset D .
$$

Thus $\lambda_{D}\left(z_{1}, z_{2}\right)=\left|z_{1}-z_{2}\right|$ and since euclidean disks in $D$ are convex with respect to the hyperbolic geometry in $D[\mathbf{J} \varnothing]$,

$$
\gamma \subset \bar{B}\left(\frac{z_{1}+z_{2}}{2}, \frac{\left|z_{1}-z_{2}\right|}{2}\right) \subset B\left(z_{1}, \frac{\operatorname{dist}\left(z_{1}, \partial D\right)}{2}\right) .
$$

Then by Lemma 1.3

$$
\min _{j=1,2} \ell\left(\gamma\left(z_{j}, z\right)\right) \leq \ell(\gamma) \leq k\left|z_{1}-z_{2}\right| \leq k \operatorname{dist}(z, \partial D)
$$

for all $z \in \gamma$ and $k$ is an absolute constant.

Next suppose that (4.7) does not hold. By compactness there exists a point $z_{0} \in \gamma$ with

$$
\operatorname{dist}\left(z_{0}, \partial D\right)=\sup _{z \in \gamma} \operatorname{dist}(z, \partial D) \text {. }
$$

Let $m$ denote the largest integer for which

$$
2^{m} \operatorname{dist}\left(z_{1}, \partial D\right) \leq \operatorname{dist}\left(z_{0}, \partial D\right)
$$

and let $y_{0}$ be the first point of $\gamma\left(z_{1}, z_{0}\right)$ with 


$$
\operatorname{dist}\left(y_{0}, \partial D\right)=2^{m} \operatorname{dist}\left(z_{1}, \partial D\right)
$$

as we traverse $\gamma$ from $z_{1}$ towards $z_{0}$. Clearly

$$
\operatorname{dist}\left(y_{0}, \partial D\right) \leq \operatorname{dist}\left(z_{0}, \partial D\right)<2 \operatorname{dist}\left(y_{0}, \partial D\right) .
$$

Let $y_{1}=z_{1}$ and choose points $y_{2}, \ldots, y_{m+1} \in \gamma\left(z_{1}, z_{0}\right)$ so that $y_{i}$ is the first point of $\gamma\left(z_{1}, z_{0}\right)$ for which

$$
\operatorname{dist}\left(y_{i}, \partial D\right)=2^{i-1} \operatorname{dist}\left(y_{1}, \partial D\right)
$$

as we traverse $\gamma$ from $z_{1}$ towards $z_{0}$. Then $y_{m+1}=y_{0}$ and let $y_{m+2}=z_{0}$.

We show first that for $i=1, m+1$

$$
\left\{\begin{aligned}
h_{D}\left(y_{i}, y_{i+1}\right) & \leq 2^{4} \cdot b^{2} \\
\ell\left(\gamma\left(y_{i}, y_{i+1}\right)\right) & \leq 2^{7} \cdot b^{2} \operatorname{dist}\left(y_{i}, \partial D\right) . .
\end{aligned}\right.
$$

Fix $i \in\{1, m+1\}$ and set

$$
t=\frac{\ell\left(\gamma\left(y_{i}, y_{i+1}\right)\right)}{\operatorname{dist}\left(y_{i}, \partial D\right)}
$$

If $z \in \gamma\left(y_{i}, y_{i+1}\right)$, then by (4.9), (4.10)

$$
\operatorname{dist}(z, \partial D) \leq \operatorname{dist}\left(y_{i+1}, \partial D\right) \leq 2 \operatorname{dist}\left(y_{i}, \partial D\right)
$$

and hence

$$
t=\int_{\gamma\left(y_{i}, y_{i+1}\right)} \frac{|d z|}{\operatorname{dist}\left(y_{i}, \partial D\right)} \leq 8 h_{D}\left(y_{i}, y_{i+1}\right)
$$

Since

$$
j_{D}^{\prime}\left(y_{i}, y_{i+1}\right) \leq \log \left(\frac{\lambda_{D}\left(y_{i}, y_{i+1}\right)}{\operatorname{dist}\left(y_{i}, \partial D\right)}+1\right) \leq \log (t+1),
$$

(4.2) implies that

$$
h_{D}\left(y_{i}, y_{i+1}\right) \leq b \log (t+1) \leq b(t+1)^{1 / 2} .
$$

If $t \geq 1$, then

$$
t \leq 8 h_{D}\left(y_{i}, y_{i+1}\right) \leq 8 b(t+1)^{1 / 2} \leq 8 b(2 t)^{1 / 2}
$$

which implies

$$
t \leq 2^{7} \cdot b^{2}
$$

and hence

$$
h_{D}\left(y_{i}, y_{i+1}\right) \leq b\left(2 \cdot 2^{7} \cdot b^{2}\right)^{1 / 2}=2^{4} \cdot b^{2} .
$$


Thus we obtain (4.11). If $t<1$, then $t \leq 2^{7} \cdot b^{2}$ and again we obtain (4.11). This completes the proof of (4.11).

Now [GP, Lemma 2.1] and (4.11) imply that for $z \in \gamma\left(y_{i}, y_{i+1}\right)$, $i=1, \ldots, m+1$

$$
\log \frac{\operatorname{dist}\left(y_{i+1}, \partial D\right)}{\operatorname{dist}(z, \partial D)} \leq 4 h_{D}\left(z, y_{i+1}\right) \leq 4 h_{D}\left(y_{i}, y_{i+1}\right)<2^{6} \cdot b^{2}=c_{0}
$$

and thus

$$
\operatorname{dist}\left(y_{i+1}, \partial D\right) \leq e^{c_{0}} \operatorname{dist}(z, \partial D) .
$$

If $z \in \gamma\left(z_{1}, z_{0}\right)$, then $z \in \gamma\left[y_{i_{0}}, y_{i_{0}+1}\right]$ for some $i_{0} \in\{1, m+1\}$ and hence by (4.10), (4.11) and (4.12)

$$
\begin{aligned}
\min _{j=1,2} \ell\left(\gamma\left(z_{j}, z\right)\right) & \leq \ell\left(\gamma\left(z_{1}, z\right)\right) \leq \sum_{i=1}^{i_{0}} \ell\left(\gamma\left[y_{i}, y_{i+1}\right]\right) \\
& \leq 2 c_{0} \sum_{i=1}^{i_{0}} \operatorname{dist}\left(y_{i}, \partial D\right)=2 c_{0}\left(2^{i_{o}}-1\right) \operatorname{dist}\left(y_{1}, \partial D\right) \\
& <2 c_{0} \operatorname{dist}\left(y_{i_{o}+1}, \partial D\right) \leq 2 c_{0} e^{c_{0}} \operatorname{dist}(z, \partial D) .
\end{aligned}
$$

Likewise, if $z \in \gamma\left(z_{2}, z_{0}\right)$, then we also have (4.13). Therefore by (4.8) and (4.13) $D$ is a $c$-John disk with $c=2 c_{0} e^{c_{0}}$.

REMARK 4.14. Theorem 4.1 is easily translated into a result for the quasihyperbolic distance in $D, k_{D}$. If we assume that quasihyperbolic geodesics are double $c$-cone arcs in $D$, the result for $k_{D}$ can be generalized to finitely connected domains in the plane, and to domains in $\mathrm{R}^{n}$ which are quasiconformal images of uniform domains. In the quasihyperbolic case, the proof of sufficiency of Theorem 4.1 shows that in a domain $D \subset \mathrm{C}$ satisfying

$$
k_{D}\left(z_{1}, z_{2}\right) \leq b j_{D}^{\prime}\left(z_{1}, z_{2}\right),
$$

quasihyperbolic geodesics are double $c$-cone arcs, where $c$ depends only on $b$.

\section{REFERENCES}

[GH] F. W. Gehring and W. K. Hayman, An inequality in the theory of conformal mapping, J. Math. Pure Appl. 9 (1962), 353-361.

[GHM] F. W. Gehring, K. Hag and O. Martio, Quasihyperbolic geodesics in John domains, Math. Scand. 65 (1989), 75-92.

[GM1] F. W. Gehring and O. Martio, Quasidisks and the Hardy-Littlewood property, Complex Variables 2 (1983), 67-78.

[GM2] F. W. Gehring and O. Martio, Lipschitz classes and quasiconformal mappings, Ann. Acad. Sci. Fenn. Ser. A I Math. 10 (1985), 203-219. 
[GM3] F. W. Gehring and O. Martio, Quasiextremal distance domains and extension of quasiconformal mapping, J. Analyse Math 45 (1985), 181-206.

[GO] F. W. Gehring and B. G. Osgood, Uniform domains and the quasihyperbolic metric, J. Analyse Math 36 (1979), 50-74.

[GP] F. W. Gehring and B. P. Palka, Quasiconformally homogeneous domains, J. Analyse Math 30 (1976), 172-199.

[HL] G. H. Hardy and J. E. Littlewood, Some properties of fractional integrals. II, Math Z. 34 (1932), 403-439.

[H] D. A. Herron, The Harnack and other conformally invariant metrics, Kodai Math. J. 10 (1978), 9-19.

[Ja] S. Jaenisch, Length distortion of curves under conformal mapping, Michigan Math. J. 15 (1968), 121-128.

[JK] D. S. Jerison and C. E. Kenig, Boundary behavior of harmonic functions in non-tangentially accessible domain, Adv. in Math. 46 (1982), 80-147.

[Jø] V. Jørgensen, On an inequality for the hyperbolic measure and its applications in the theory of functions, Math. Scand. 4 (1956), 113-124.

[KW] R. Kaufman and J.-M. Wu, Distances and the Hardy-Littlewood property, Complex Variables 4 (1984), 1-5.

[K] K. Kim, Necessary and sufficient conditions for the Bernstein inequality, Ann. Acad. Sci. Fenn. Ser. A I Math. 20 (1995), 419-432.

[Kr] J. G. Krzyż, Quasicircles and harmonic measure, Ann. Acad. Sci. Fenn. Ser. A I Math. 12 (1987), 19-24.

[MS] O. Martio and J. Sarvas, Injectivity theorems in plane and space, Ann. Acad. Sci. Fenn. Ser. A I Math. 4 (1978), 383-401.

[NV] R. Näkki and J. Väisälä, John disks, Exposition. Math. 9 (1991), 3-43.

[P] C. Pommerenke, One-sided smoothness conditions and conformal mappings, J. London Math. Soc. 26 (1982), 77-82.

[PR] C. Pommerenke and S. Rohde, The Gehring-Hayman inequality in conformal mapping, Quasiconformal Mappings and Analysis, Springer-Verlag, submitted.

[R] K. Kim. Ryu, Properties of John disks, University of Michigan Ph.D. Thesis, University of Michigan (1991).

[V] J. Väisälä, Lectures on n-dimensional quasiconformal mappings, Lecture Notes in Math. 229, 1971.

DEPARTMENT OF MATHEMATICS

PUSAN WOMEN'S UNIVERSITY

PUSAN

617-736 KOREA
DEPARTMENT OF MATHEMATICS

UNIVERSITY OF MICHIGAN

ANN ARBOR, MI 48109

USA 